\title{
Intergenomic translocations of polyploid oats (genus Avena) revealed by genomic in situ hybridization
}

\author{
Mikako Hayasaki, Toshinobu Morikawa* and Isao Tarumoto \\ Graduate School of Agriculture and Biological Sciences, Osaka Prefecture University, \\ Sakai, Osaka 599-8531 Japan
}

(Received 21 March 2000, accepted 29 June 2000)

\begin{abstract}
Wild and cultivated hexaploid oats share the same genomes (AACCDD) and display a considerable level of interspecific variation in both plant and chromosome morphology. The GISH was utilized to detect the interspecific genomic compositions in four hexaploid and two tetraploid oats using total genomic DNA of Avena eriantha (a C-genome diploid) as probe. Intergenomic translocations between $\mathrm{A} / \mathrm{D}$ and C-genome chromosomes were frequently observed in hexaploid and tetraploid species. In the hexaploid, two pairs of A/D genome segments on C-genome chromosome (A/D-C) translocation and four to six pairs of C-genome segments on A/D genome chromosome $(\mathrm{C}-\mathrm{A} / \mathrm{D})$ translocation were clearly identified whilst the number of A/D-C translocations was constant among species. In the tetraploid A. maroccana (AACC), a pair of A-C and four pairs of C-A translocations were observed. Moreover, the $\mathrm{A} / \mathrm{D}$ translocation segments on chromosome $5 \mathrm{C}$ was detected only in A. byzantina and A. maroccana, whilst A/D-C translocations were observed on the $1 \mathrm{C}$ and $7 \mathrm{C}$ of A. sativa, A. fatua and A. sterilis. A. byzantina did however also carry the $1 \mathrm{C}$ rearrangement. This result shows that $A$. byzantina has retained a similar genomic constitution to the tetraploid ancestor of hexaploid oats, A. maroccana. Three pairs of A-C translocations were detected only in A. murphyi (AACC), and two pairs of those were the $1 \mathrm{C}$ and $7 \mathrm{C}$ as well as the three hexaploid species except $A$. byzantina.
\end{abstract}

\section{INTRODUCTION}

The genus Avena L. (Poaceae) comprises a polyploid series of diploid, tetraploid and hexaploid species with a basic chromosome number of seven. Based on chromosome number, genome constitution and morphology of the diaspore, thirty taxonomic species of this genus have been classified into 11 genomic groups (Leggett, 1992). The wild and cultivated hexaploid oats, A. sativa, A. byzantina, A. fatua and A. sterilis, are interfertile and occasionally cross in nature. Because of these close genetical and chromosomal affinities, these species are considered to belong to the a single biological species (Ladizinsky and Zohary, 1971) with the genomic designation AACCDD (Rajhathy and Thomas, 1974).

The identification of individual chromosomes of hexaploid oats has been reported using classical meiotic chromosome pairing and somatic karyotype studies (Rajhathy and Thomas, 1974; Nishiyama et al., 1989). By using the

Edited by Takashi Endo

* Corresponding author. E-mail: morikawa@plant.osakafu-u.ac.jp
C-banding analysis, a technique preferentially staining heterochromatin, the C-genome chromosomes were distinguished from the $\mathrm{A}$-and $\mathrm{D}$-genome chromosomes (Linares et al., 1992; Jellen et al., 1993), and the latter authors demonstrated C-banding polymorphisms among the hexaploid species studied.

Recently, genomic in situ hybridization (GISH) with fluorescent dye has proven to be a very useful tool for allocating chromosomes to genomes in allopolyploid species (Mukai et al. 1993). At the moment, the GISH was unable to separate the A- and D-genome chromosomes in hexaploid oats. But absolute discrimination between A/D- and C-genome chromosomes has been demonstrated by several oat workers (Jellen et al., 1994; Chen and Armstrong, 1994; Leggett and Markhand, 1995; Yang et al., 1999). These authors observed small C-genome segments translocated onto A- or D-genome chromosomes (abbreviated as C-A/D translocation) and vice versa (abbreviated as A/D-C) for A- or D-segments translocated onto $\mathrm{C}$-genome chromosomes. The variation of number and position of the intergenomic translocations has been observed to be in parts of hexaploid and tetraploid genotypes. 

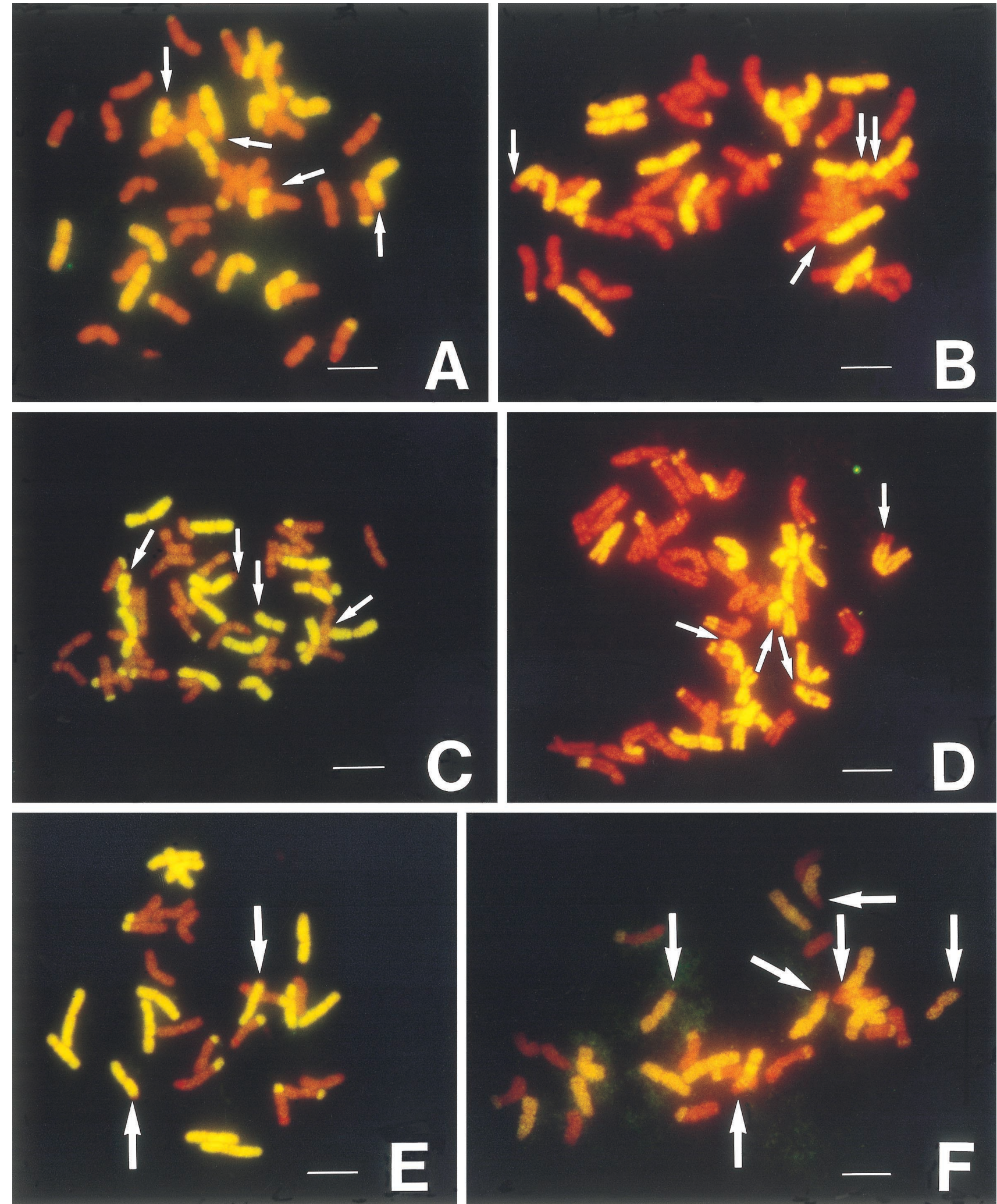

Fig. 1. Genomic in situ hybridization with biotinylated A. eriantha DNA to chromosomes of A. sativa (A), A. byzantina (B), A. fatua (C), A. sterilis (D), A. maroccana (E) and A. murphyi (F). Hybridization signals are visualized in yellow. The arrows show the A/D-C translocations. Scale bar is $10 \mu \mathrm{m}$. 
So, it is possible to estimate the genomic evolution from the possible tetraploid ancestors to all the hexaploid species and intra-hexaploid differentiation depending on the number and position of the intergenomic translocations revealed by GISH.

The present paper reports interspecific genomic translocations in four hexaploid (AACCDD) and two tetraploid (AACC) species having C-genome revealed by the GISH and discusses the genomic composition and chromosome evolution of polyploid oats.

\section{MATERIALS AND METHODS}

Plant materials Two cultivated hexaploid (A. sativa L. 'Sun II' and A. byzantina C. Koch 'Kanota'), two wild hexaploid (A. fatua accession 50-10 and A. sterilis accession 69-15) and two wild tetraploid oats (A. maroccana accession $69-14$ and $A$. muphyi accession $74-17$ ) were used for collecting root tips for chromosome preparations of the GISH. The C-genome diploid species, A. eriantha accession 69-5 was used for isolating total C-genomic DNA's for preparation of probe.

Chromosome preparation Chromosome preparations were carried out with a modification of the procedure of Matsuda and Kishigami (1981). Root tips were pretreated in ice water for $24 \mathrm{~h}$ at $0^{\circ} \mathrm{C}$, fixed in Farmer's fixative (ethyl alcohol: acetic acid=3:1), and dehydrated through descending ethanol series. The root tips were then digested in the mixture of $0.5 \%$ Pectolyase Y-23 (Kikkoman) and $0.5 \%$ Cellulase "ONOZUKA" RS (Yakult) at $37^{\circ} \mathrm{C}$ for $50 \mathrm{~min}$. Meristematic regions of the root tips were spread on a slide glass in a few drops of Farmer's fixative and then air-dried.

In situ hybridization Hybridization and detection were carried out with a modification of the procedure of Mukai et al. (1993) and Schwarzacher et al. (1989). The probe DNA from A. eriantha was labelled by nick translation with biotin-14-dATP using the BioNick Labeling System (GIBCO/BRL). After hybridization, the signals were detected by fluorochrome fluorescein isothiocyanated avidin (FITC) and biotinylated anti-avidin-D. The slides were doubly stained with propidium iodide (PI) and 4,6diamidino-2-phenylindole (DAPI). The FITC-avidin hybridization signals and PI are detected by their yellow and red fluorescence. Photographs were taken with Kodak Ektachrome ISO 400 color slide film.

Karyotype analysis The seven chromosomes belonging to the C-genome were identified by observing at least fifty good figure cell plates. According to chromosome length, each C-genome chromosome was numbered from the longest $1 \mathrm{C}$ to the shortest $7 \mathrm{C}$ chromosomes. And, the arm ratio was carefully measured to clarify the difference of each chromosome. It was very easy to distinguish the longest, intermediate and shortest chromosomes, for instance, $1 \mathrm{C}, 5 \mathrm{C}$ and $7 \mathrm{C}$.

\section{RESULTS}

Hexaploid species The GISH was performed using total genomic DNA derived from the C-genome diploid $A$. eriantha as probe to examine the $\mathrm{C}$-genome chromosome structures in four hexaploid oats. Seven pairs of the C-genome chromosomes were successfully labelled and the remaining 14 pairs of chromosomes were unlabelled over all the hexaploid species indicating that these were A- or D-genome derived chromosomes (Fig.1-A,B,C and D). Moreover, the hybridization sites also clearly indicated the A/D-C and C-A/D intergenomic translocations. And the positions of all the translocations were at the terminal regions of the long arms of chromosomes.

Two pairs of C-genome chromosomes carrying an A/Dgenome translocation were observed in all the hexaploid species, but different chromosomes were involved depending on the species. Based on karyotype analysis, the A/ D-C translocations were located in terminal regions of the long arm of $1 \mathrm{C}$ and $7 \mathrm{C}$ of A. sativa, A. fatua and A. sterilis whilst in A. byzantina chromosome 7C did not carry a translocation but 5C did (Fig.2-A,B,C and D).

The number of C-A/D translocations also differed among the hexaploid species, four pairs being observed in $A$.
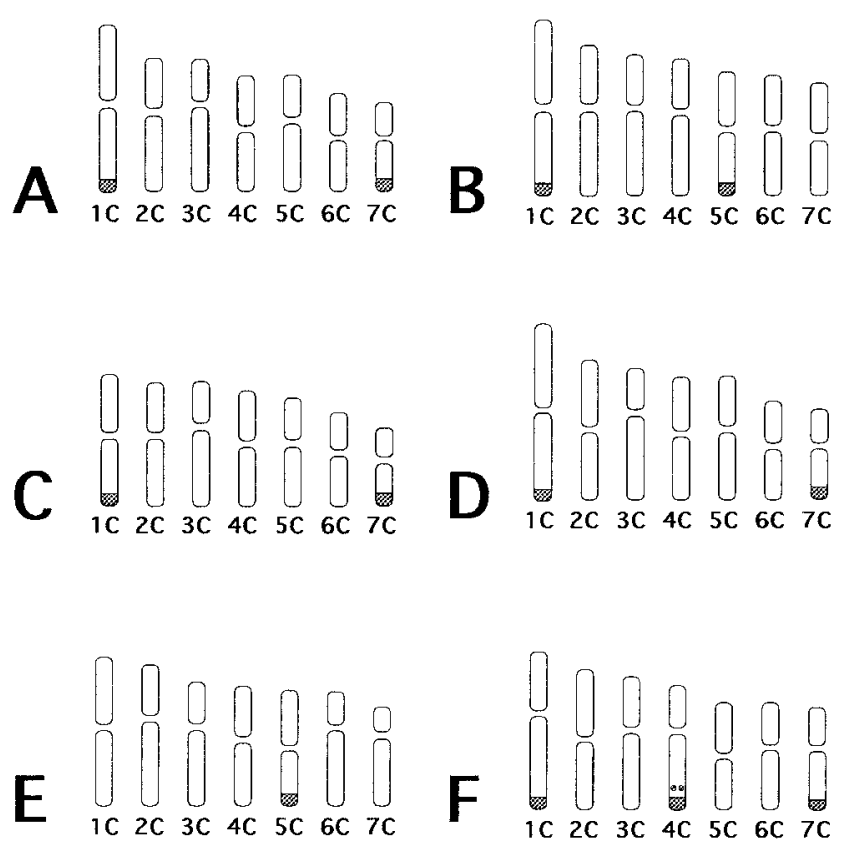

Fig. 2. Comparison of the $\mathrm{C}$-genome chromosomes of $A$. sativa (A), A. byzantina (B), A. fatua (C), A. sterilis (D), A. maroccana (E) and $A$. murphyi (F), showing positions of intergenomic translocation sites shadowed. 
Table 1. The genomic constitution of four hexaploid (AACCDD) and two tetraploid (AACC) of Avena species.

\begin{tabular}{|c|c|c|c|c|c|c|}
\hline \multirow[b]{2}{*}{ Species } & \multirow[b]{2}{*}{ Ploidy } & \multicolumn{2}{|c|}{ No. of intact chromosomes } & \multicolumn{3}{|c|}{ No. of intergenomic translocations } \\
\hline & & A/D-genome & C-genome & $\mathrm{C}$ on $\mathrm{A} / \mathrm{D}$-gemome & $\mathrm{A} / \mathrm{D}$ on $\mathrm{C}$-genome & Total \\
\hline A. sativa & $6 \mathrm{X}$ & 18 & 10 & 10 & 4 & 14 \\
\hline A. byzantina & $6 \mathrm{X}$ & 20 & 10 & 8 & 4 & 12 \\
\hline A. fatua & $6 \mathrm{X}$ & 18 & 10 & 10 & 4 & 14 \\
\hline A. sterilis & $6 \mathrm{X}$ & 16 & 10 & 12 & 4 & 16 \\
\hline A. maroccana & $4 \mathrm{X}$ & 6 & 12 & 8 & 2 & 10 \\
\hline A. murphyi & $4 \mathrm{X}$ & 6 & 8 & 8 & 6 & 14 \\
\hline
\end{tabular}

byzantina, five pairs in A. sativa and A. fatua, and six pairs in A. sterilis.

Tetraploid species In two tetraploid species, seven pairs of C-genome chromosomes were clearly labelled with total DNA's of A. eriantha and seven pairs of A-genome chromosomes were unlabelled (Fig. 1-E and F). The tetraploid species also had the same intergenomic translocations as the hexaploids. A. maroccana had a pair of $\mathrm{A} / \mathrm{D}-\mathrm{C}$ and four pairs of C-A/D translocations in terminal regions of chromosomes. However, A. murphyi had three pairs of A/D-C and four pairs of C-A/D translocations in both terminal and interstitial regions of chromosomes. The GISH plate of $A$. murphyi is presented here for the first time. The A/D-C translocation sites differed between the tetraploid species, a pair (5C) observed in $A$. maroccana (Fig.2-E) and three pairs (1C, 4C and 7C) in A. murphyi (Fig.2-F).

Number of A/D-C and C-A/D translocations The genomic constitution of the four hexaploid and two tetraploid species was summarized in Table 1 . The A/D-genome chromosomes carried two to four times as many intergenomic translocation segments as the $\mathrm{C}$-genome. The number of C-genome chromosome carrying translocated $\mathrm{A} / \mathrm{D}$ genome segment was constant in hexaploid but varied in tetraploid species. The maximum total number of translocations observed was eight pairs in A. sterilis and the minimum was five pairs in A. maroccana.

\section{DISCUSSION}

The GISH technique allowed us to detect intergenomic translocations between A/D- and C-genome chromosomes in polyploid oats. And it seems reasonable to expect that intergenomic translocations between the A- and D-genome chromosomes also occur (Leggett and Markhand, 1995). Clearly, since oats are intrinsically an inbreeding species, natural populations are likely to have relatively stable genomes. However, the small proportion of outcrossing which is known to occur (1-2\%) in oats, could give favourable environmental conditions, quite rapidly lead to karyotype restructuring. A number of authors have at- tempted to identify individual C-genome chromosomes in hexaploid oats by GISH and FISH (e.g. Chen and Armstrong, 1994; Jellen et al., 1994; Leggett and Markhand, 1995; Yang et al., 1999). Fominaya et al. (1995) and Linares et al. (1996) performed FISH analysis using a repeated DNA sequence (pAm1) specific to C-genome chromosomes. When total genomic DNA from $A$. eriantha was used in this study, one to three pairs of Cgenome chromosomes carrying A/D translocations were detected in four hexaploid and two tetraploid oats. In contrast, using pAm1, Linares et al. (1996) reported that it was possible to detect the $\mathrm{A} / \mathrm{D}$ translocations on almost all the $\mathrm{C}$-genome chromosomes in three hexaploid oats. Most of the translocations observed in Avena was detected on terminal position of chromosome which is rich with telomere repeat sequences. Thus, in the FISH analysis, the repetitive pAm 1 probe detected much more translocations than total genomic DNA of $A$. eriantha because that the pAm1 probe involved a lot of variable flanking regions, like microsatellites.

Jellen et al. (1994) reported two pairs of A/D-C translocations whilst Chen and Armstrong (1994) and Leggett and Markhand (1995) reported three pairs in A. sativa. These translocations were usually located on the long arms of the chromosome. Though Jellen et al. (1994) reported a pair of $\mathrm{A} / \mathrm{D}$ translocations to be located at an interstitial position on the long arm of a C-genome chromosome in A. sativa. The results of this study correspond to those of Jellen et al. (1994) except for that we did not observe an interstitial translocation in the hexaploid species but in the tetraplod, A. murphyi (Fig. 2-F-4C). In this study, the terminal A/D translocation segments of $A$. byzantina were identified as on the chromosomes $1 \mathrm{C}$ and 5C whilst Jellen et al. (1994) identified the rearrangements on the $1 \mathrm{C}$ and 7C. The A/D-C translocation on the $5 \mathrm{C}$ appears to be unique to $A$. byzantina and the tetraploid, A. maroccana, although the degree of chromosome homology could not be confirmed. We observed that the terminal A/D translocations in A. fatua occurred on the $1 \mathrm{C}$ and 7C. Yang et al. (1999) reported that the terminal $\mathrm{A} / \mathrm{D}-\mathrm{C}$ translocations on the $1 \mathrm{C}, 3 \mathrm{C}$ or $4 \mathrm{C}$, and in addition, detected a terminal A/D translocation on each arm of chromosome 7C. In our study, a terminal A/D translocations in A. sterilis was observed on chromosomes $1 \mathrm{C}$ and $7 \mathrm{C}$ and 
corresponded to the results of Jellen et al. (1994). Evidently the number of chromosome rearrangements observed in polyploid oats is dependent on species, accession and the detection technique used.

Although both tetraploid species, A. maroccana and A. murphyi were detected the translocation segment on medium length chromosome of seven pairs of C-genome chromosomes, two hexaploid species, A. byzantina (our result) and A. fatua (Yang et al., 1999) were also detected on medium length chromosome. It suggests that the chromosomes with the segment of two hexaploids are conservative. We considered that the $7 \mathrm{C}$ chromosome was derived from structural change of medium C-genome chromosome. To prove this hypothesis, C-banding or FISH method using repetitive DNA probes such as 5S-rDNA and 18S-rDNA are needed to identify the numbers of $\mathrm{C}$-genome chromosome clearly, and to analyze the chromosome homology between the related species.

The intergenomic translocations were not occurred reciprocally between $\mathrm{C}$ - and A/D-genomes. These translocations also do not lead to multiple chromosome pairings at meiosis, but lead to diploidization of ployploid oats. After that, a genetic control system suppressing multivalent formation was needed to keep a new stable genotype. The genomes of polyploid oats were remarkably reorganized through the intergenomic translocations. The hexaploid oat is a typical allopolyploid species consisted with three genomes as well as hexaploid wheat. After polyploidization, however, mode of genomic evolution of oats was quite different from that of wheat.

Most of the chromosome rearrangements observed in both hexaploid and tetraploid oats involved the segments of C-genome chromosomes translocated onto A- or Dchromosomes. In additions, the $\mathrm{C}$-genome chromosome segments increased in the hexaploid species (Table 1). This result suggests that the $\mathrm{C}$-genome is changeable and has the tendency for heterochromatin of the C-genome to be transferred to the terminal regions of the $\mathrm{A} / \mathrm{D}$-genome chromosomes. The karyotype of the $\mathrm{C}$-genome in hexaploid species consists of median and submedian chromosomes, whilst in the tetraploid they are median and subtelocentric chromosomes, the diploid species consisting of subtelocentric chromosomes (Hayasaki unpublished data). These diverse karyotypes of the $\mathrm{C}$-genome at the different ploidy levels might well result from the chromosomal translocations from the C-genome to the A- or Dgenome during speciation.

The authors are grateful to Dr. J. M. Leggett (Institute of Grassland and Environmental Research, Plas Gogerddan, U.K.) for his critical reading of the manuscript and encouraging suggestions. The research was financially supported by the Sasagawa Scientific Research Grant from The Japan Science Society.

\section{REFERENCES}

Chen, Q. and Armstrong, K. (1994) Genomic in situ hybridization in Avena sativa. Genome 37, 607-612.

Fominaya, A., Hueros, G., Loarce, Y. and Ferrer, E. (1995) Chromosomal distribution of a repeated DNA sequence from $\mathrm{C}$-genome heterochromatin and the identification of a new ribosomal DNA locus in the Avena genus. Genome 38, 548557.

Jellen, E. N., Phillips, R. L. and Rines, H. W. (1993) C-banded karyotypes and polymorphisms in hexaploid oat accessions (Avena spp.) using Wright's stain. Genome 36, 1129-1137.

Jellen, E. N., Gill, B. S. and Cox, T. S. (1994) Genomic in situ hybridization differentiates between $\mathrm{A} / \mathrm{D}$ - and C-genome chromatin and detects intergenomic translocation in polyploid oat species (genus Avena). Genome 37, 613-618.

Ladizinsky, G. and Zohary, D. (1971) Notes on species delimination, species relationships and polyploidy in Avena L. Euphytica 20, 380-395.

Leggett, J. M. (1992) Classification and speciation in Avena. In Oat Science and Technology-Agronomy Monograh no. 33, 2952 .

Leggett, J. M. and Markhand, G. S. (1995) The genomic identification of some monosomics of Avena sativa L. cv. Sun II using genomic in situ hybridization. Genome 38, 747-751.

Linares, C., Vega, C., Ferrer, E. and Fominaya, A. (1992) Identification of C-banded chromosomes in meiosis and the analysis of nucleolar activity in Avena byzantina C. Koch cv. 'Kanota'. Theor. Appl. Genet. 83, 650-654.

Linares, C., Gonzalez, J., Ferrer, E. and Fominaya, A. (1996) The use of double fluorescence in situ hybridization to physically map the positions of $5 \mathrm{~S}$ rDNA genes in relation to the chromosomal location of 18S-5.8S-26S rDNA and a C genome specific DNA sequence in the genus Avena. Genome 39, 535542.

Matsuda, T. and Kishigami, N. (1981) Electron microscopic ammonical silver reaction for basic nuclear proteins of Dumoriera hirsuta, Liverwort. Sci. Rep. Yokohama Nat. Univ., Sec. II 28, 11-18.

Mukai, Y., Nakahara, Y. and Yamamoto, M. (1993) Simultaneous discrimination of the three genome in hexaploid wheat by multicolor fluorescence in situ hybridization using total genomic and highly repeated DNA probes. Genome 36, 489494.

Nishiyama, I., Yabuno, T. and Taira, T. (1989) Genomic affinity relationships in the genus Avena. Plant Breeding 102, 2230 .

Rajhathy, T. and Thomas, H. (1974) Cytogenetics of oat (Avena L.). Misc. Publ. Genet. Soc. Can. No 2. The Genetics Society of Canada, Canada.

Schwarzacher, T., Leitch, A.R., Bennett, M.D. and HeslopHarrison, J. S. (1989) In situ localization of parental genomes in wide hybrid. Ann. Bot. 64, 315-324.

Yang, Q., Hanson, L. Bennett, M.D. and Leitch, I.J. (1999) Genome structure and evolution in the allohexaploid weed Avena fatua L. Genome 42, 512-518. 